\title{
Is subcranial Le Fort III plus Le Fort I osteotomy stable? ${ }^{\text {ir }}$
}

\author{
Fernanda Brasil Daura Jorge Boos Lima ${ }^{a}$, Eduardo Hochuli Vieira b, Philipp Juergens ${ }^{\text {, }}$ \\ Sergio Monteiro Lima Junior ${ }^{\mathrm{a}, \mathrm{d}, \text { * }}$ \\ a Oral and Maxillofacial Surgery, Federal University of Minas Gerais, Belo Horizonte, Brazil \\ b Oral and Maxillofacial Surgery, State University of São Paulo, Araraquara, Brazil \\ ${ }^{c}$ Department of Oral \& Maxillofacial Surgery, University Hospital Basel, Faculty of Medicine, University of Basel, Basel, Switzerland \\ ${ }^{\mathrm{d}}$ MaterDei Healthcare Network, Division of Oral and Maxillofacial Surgery, Brazil
}

\section{A R T I C L E I N F O}

\section{Article history:}

Paper received 17 April 2017

Accepted 11 September 2017

Available online 3 October 2017

\section{Keywords:}

Orthographic surgery

Le Fort III osteotomy

Stability

\begin{abstract}
A B S T R A C T
The purpose of this study was to test whether associated subcranial Le Fort III (sLF III) and Le Fort I (LF I) osteotomies are stable after large advancements of the middle third of the face and maxilla. The authors designed a retrospective study and enrolled a sample of consecutive patients with midface hypoplasia treated with associated SLF III and LF I osteotomies in this IRB-approved study between September 2013 and February 2015. To test whether the long-term stability was satisfactory, the authors compared cephalometric changes from immediately after surgery to 18 months after surgery taken from multi-slice computed tomography using two different third-party imaging software programs. Statistical significance was set as $P \leq 0.05$. The sample comprised 11 patients (mean age $23.84 \pm 4.17 \mathrm{yr} ; 54 \% \mathrm{men}$ ). The mean advancement of the upper incisor immediately after surgery was $10.03 \pm 1.6 \mathrm{~mm}$. After $18 \mathrm{months}$, the position of the upper incisor did not vary significantly $(10.18 \pm 2.35 \mathrm{~mm})$. All other cephalometric landmarks did not present statistically significant differences between immediately after and 18 months after surgery, with horizontal and vertical variations of less than one millimetre. This study supports that SLF III and LF I osteotomies are effective in maintaining stable horizontal and vertical skeletal positioning after surgery.
\end{abstract}

๑ 2017 European Association for Cranio-Maxillo-Facial Surgery. Published by Elsevier Ltd. All rights reserved.

\section{Introduction}

The underdevelopment of midface hypoplasia usually causes severe exorbitism, obstructive sleep apnoea, distorted facial appearance, and class III malocclusion (Bouw et al., 2015). The treatment of a severely hypodeveloped midface is with a Le Fort III osteotomy (LF III) or an osteogenic distraction with a LF III. The conventional LF III osteotomy constitutes a one-step technique in which the midfacial segment is osteotomised, advanced, and fixed in the desired position with bone grafts. The subcranial Le Fort IIII (sLF III) osteotomy is a technique designed to advance the midface without the need of bone grafts to improve stability, because there

\footnotetext{
tr Received from Universidade Federal de Minas Gerais, Department of Clinics, Pathology and Surgery, Division of Oral and Maxillofacial Surgery, Belo Horizonte, Brazil.

* Corresponding author. Faculdade de Odontologia, Departamento de Clínica, Patologia e Cirurgia, Universidade Federal de Minas Gerais, Campus Pampulha Avenida Antônio Carlos 6627, Pampulha, Belo Horizonte, MG CEP 31270-901, Brazil.

E-mail addresses: limajrsm@hotmail.com, limajrsm@ufmg.br (S.M. Lima Junior).
}

is a large amount of contact between segments, as opposed to the classic technique, in which a higher osteotomy and grafts are needed at the orbit and zygoma (Cheung et al., 2010). Usually, a LF I osteotomy is performed at the same surgical time as the LF III to obtain a greater advancement of the upper incisor and correct the occlusal plane.

The LF III osteotomy was classified and derived from Tessier's reports (Tessier, 1967, 1971a, 1971b). Other authors have indicated LF III osteotomies for syndromic patients with several forms of craniofacial dysostosis, because of the physiological and functional problems associated with these disorders (Epker and Wolford, 1975; Tiwana and Turvey, 2004). Currently, to treat severe discrepancies and achieve an adequate intermaxillary relationship and a stable occlusion, LF III osteotomy in combination with LF I osteotomy is indicated for the treatment of syndromic and nonsyndromic patients presenting with severe class III malocclusion and $10 \mathrm{~mm}$ or more of reverse overjet (Tiwana and Turvey, 2004; Nout et al., 2008).

There are many reports in the literature discussing the stability of LF III osteotomy (Kaban et al., 1986; Bachmayer and Ross, 1986; 
Ousterhout et al., 1986; McCarthy et al., 1990; Schmitz et al., 1995; Meazzini et al., 2005; Shetye et al., 2010a, 2010b). The LF III advancement is stable, but there are differences in the techniques used, especially concerning recommended bone grafts for stable fixation in most of the studies. To the best of our knowledge, only one study has discussed the stability of a sFL III osteotomy and reported that the degree of horizontal stability and the forward direction of postoperative skeletal changes are consistent with previously published results of LF III osteotomies (Tiwana et al., 2000).

It is important to understand the stability of a given surgery, how much change can be seen as a result of large advancements, and how the results will change over time. sLF III osteotomies, although widely accepted, are not widely performed. Therefore, long-term results are an important contribution to guide treatment and indicate such procedures.

The purpose of this study was to evaluate the long-term stability (18 months) in a series of non-syndromic patients who underwent sLF III osteotomy in combination with LF I osteotomy for the improvement of midface deficiency by superimposition of computed tomography (CT) images preoperatively, immediately postoperatively, and at 18 months after surgery. The authors hypothesise that advancing the upper incisor more than $10 \mathrm{~mm}$ by sLF III and LF I osteotomies is stable in long-term evaluation. The specific aims of this study were to measure and compare the horizontal and vertical changes of the upper incisor and other middle third cephalometric points after large advancements of the middle third of the face by superimposing sequential CT images.

\section{Material and methods}

\subsection{Study design}

To address the research purpose, the investigators designed and implemented a retrospective study. The study population was composed of patients presenting for the evaluation and management of dentofacial deformities to the department of Oral and Maxillofacial Surgery between September 2013 and February 2015. The study protocol obtained local ethics review board approval under the number 56390815.5.0000.5416.

\subsection{Sample identification and selection}

To be included in the study sample, patients had to be adults; present clinically identifiable hypoplasia of the middle third of the face, including the maxilla, orbits, zygoma, and nose; and present a class III angle malocclusion, with an overjet greater than $10 \mathrm{~mm}$, measured clinically. All patients signed an informed consent agreeing to use their data in the study and underwent sLF III osteotomy with LF I osteotomy to further advance the upper incisor and correct the occlusal plane, along with sagittal split osteotomy to correct a severe class III malocclusion associated with a nasomaxillary-zygomatic deficiency. Exclusion criteria included cleft and/or syndromic patients, lack of CT data, lack of clinical followup, and growing patients.

\subsection{Surgical technique}

LF III osteotomy was performed by a standardised coronal approach toward the nasal bones, orbit cavity, zygomatic arch, and zygoma body. Bilateral endoaural extension of the coronal incision was performed to completely expose the zygomatic arch during dissection, if necessary. A sliding osteotomy was performed from the body of the zygoma to the orbit, below the lateral canthus. This sliding osteotomy was an oblique cut made with a reciprocating saw or a piezoelectric device from a point as far down and posterior as possible at the zygoma body to the lateral orbital rim, below the lateral orbital tendon attachment. These bone cuts are made bilaterally, and it is important that they are parallel and as horizontal as possible. The orbital floor is accessed through the coronal approach. The orbital floor is then separated from the skull using a piezoelectric device. This cut is made from the end of the lateral orbital rim osteotomy, $03 \mathrm{~mm}$ behind the orbital rim, extending medially until it stops behind the nasolacrimal duct. The third osteotomy was to separate the nasal bones from the skull. Care must be taken to cut the nose below the cribriform plate. A plane osteotomy, preferably parallel to the osteotomies of the zygoma body, is done to help the adaption of the cuts after advancement. The fourth osteotomy aims to separate the medial orbital wall. This cut should also be done with piezoelectric tips. This osteotomy begins at the end of the nasal bone osteotomy and continues in an inferior direction behind the lacrimal apparatus and medial tendon of the orbit until it reaches the orbital floor osteotomy. Pterygomaxillary disjunction is followed by an osteotomy from the end of the zygoma body osteotomy to the pterygoid plates. The separation of the nasal septum from the skull base is done with a septal osteotome.

After downfracture and adequate mobilisation, a surgical occlusal splint with the predetermined middle third advanced position planned is placed, and temporary intermaxillary fixation (IMF) is done. Two plates are placed bilaterally in the zygomatic bones and one plate in "Y" design achieves stabilization of the nasal bones.

A conventional LF I osteotomy follows the LF III osteotomy. The LF I can be advanced and is used to correct occlusal plane alterations. It should be kept in mind that the final vertical position of the upper incisor is obtained using the external reference at this point. No attempt should be made to correct vertical deficiencies during LF III osteotomies. After applying fixation at the maxilla, mandible sagittal split osteotomies are performed to obtain final occlusion and fixed using the hybrid technique. The patient is usually discharged after 5 days of hospitalisation. A similar surgical technique for LF III osteotomy was published by Cheung et al. (2010). All osteotomies were fixed using a standard $2.0-\mathrm{mm}$ system, except for the nasal osteotomy, which was fixed using a 1.5mm system.

\subsection{Variables}

\subsubsection{Predictor variables}

The primary predictor variable of this research was the followup period after surgery. Multi-slice CT scans were obtained 7 days before surgery (T0), $24 \mathrm{~h}$ after surgery (T1), and 18 months after surgery (T2). Multi-slice scans were made using a standardised scanning protocol, with a slice increment of $0.625 \mathrm{~mm}$, in a field of $17 \mathrm{~mm}$, at $120 \mathrm{Kv}$ and $160.60 \mathrm{~mA}$ (GE Healthcare Optima CT660 128-slice). All patients were asked to occlude teeth and rest the tongue in a relaxed position, breathe lightly, and avoid motor reaction. All scans were performed with the patients in the supine position, with a headgear to hold the head.

The three-dimensional (3D) images were constructed using Dolphin (group 1) and Mimics (group 2) and superimposed using at least 5 points of superimposition: the crista galli, the frontozygomatic sutures bilaterally, the cephalometric point zygio, at the zygomatic arch, and bilaterally. None of these points had variation before and after surgery. Both software programs had the tools for registration and superimposition. The result of superimposition was verified by the correct overlay of the 3D skulls of the sphenoid bone at coronal slices (Fig. 1), and frontal bone at axial and sagittal images. 


\subsubsection{Outcome variables}

The primary outcome variables of this study were the horizontal and vertical changes of the following cephalometric landmarks between T1 and T2: tip of the right upper incisor, the posterior nasal spine, the A-point, the right and left infra-orbital points, and tip of the nasal bones. Gender, age, pre-surgery inverted overjet, and type of surgery were recorded.

Before the stability evaluation, all databases (T0, T1, and T2) were assessed and the patient's skull was reoriented to the Frankfort horizontal (FH) using the following guidelines: (1) In the frontal view, the midsagittal plane was fixed through the centre of the midsagittal maxillary suture, and the axial plane was constructed through both infraorbital skeletal landmarks. (2) In the right sagittal view, the axial plane was placed through the right portion and right infraorbital landmarks. For standardisation, the left sagittal view was not processed to avoid orientation problems due to asymmetrically positioned porions. (3) In the transversal view (patient facing down), the midsagittal plane was constructed through crista galli and basion. In the opposite transversal view (patient facing up, or exocranial view), it was ensured that no "yaw" (transversal rotation) of the mandible or the zygomatic arches was present. A similar methodology for head orientation was published elsewhere for different purposes (Guijarro-Martínez and Swennen, 2013).

In the sagittal view, at midline, a plane parallel to $\mathrm{FH}$, passing through the sella point, was used as a horizontal reference to measure linear vertical changes. A perpendicular plane to $\mathrm{FH}$, passing through sella point, was used as a vertical reference to measure horizontal changes. The cephalometric points were measured at the three different times (T0, T1, and T2) for horizontal and vertical changes by using semi-transparent slices of superimposed CT images. Fig. 2 shows the superimposition using Dolphin software, and Fig. 3 shows the superimposition using Mimics software.

The secondary outcome variable was the data processing and measurements using two different commercially available thirdparty software programs (Dolphin Imaging1, version 11.0, Chatsworth, CA, USA and Mimics, version 16.0, Materialise, Leuven, Belgium).

\subsubsection{Other variables}

The other study variables were grouped in the following sets of variables:

Demographics. Demographic parameters were gender and age at the time of surgery.

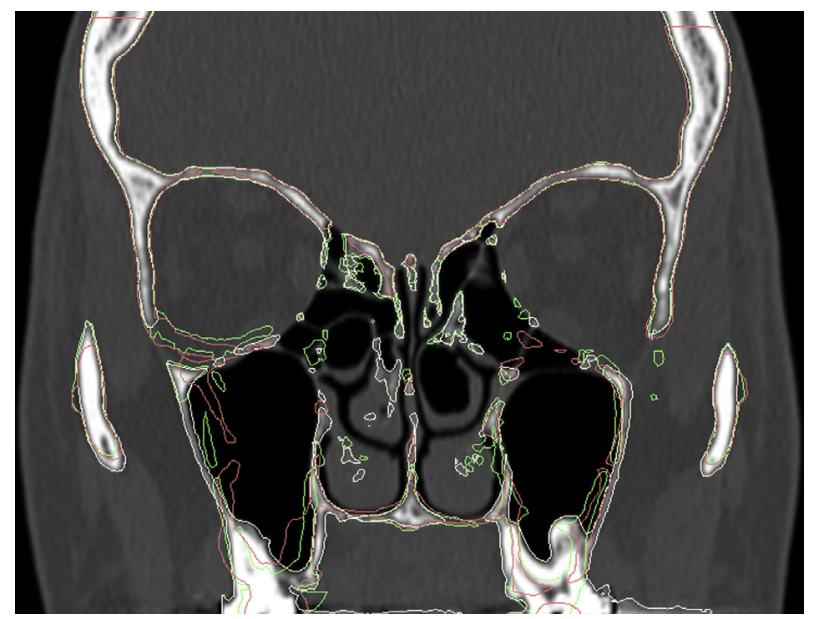

Fig. 1. Superimposition of the 3D skulls at the sphenoid bone at coronal slice.

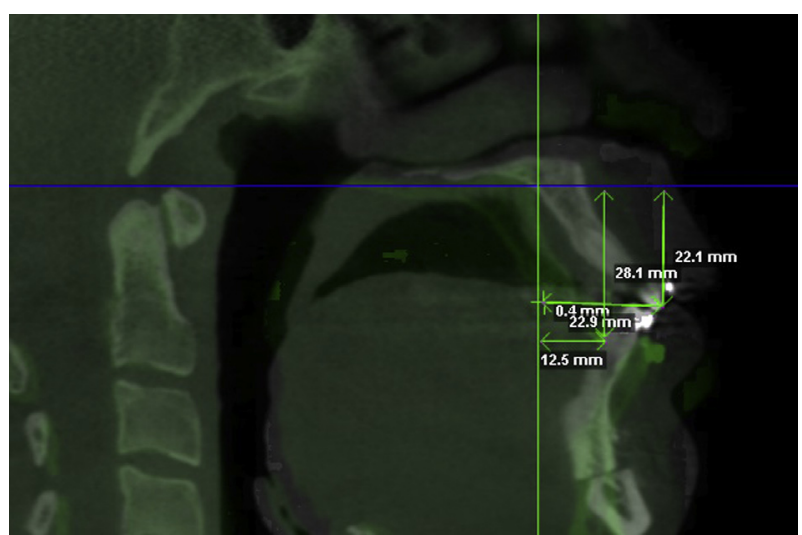

Fig. 2. Linear horizontal and vertical measures after registration and superimposition with Dolphin software.

Type of surgery. Surgical technique according to international standard clinical descriptions. Subcranial Le Fort III, Le Fort I, bilateral sagittal split osteotomy, chin osteotomy.

Pretreatment characteristics. These variables include the pretreatment overjet and the inclination of the right upper incisor in relation to the palatine bone before surgery.

\subsection{Data analysis}

The results of continuous variables were reported as means with standard deviations (SD), and categorical variables as proportion and percentages. Bivariate analysis was computed to measure the association between the primary predictor variable (follow-up) and the primary outcome variable (stability). Regressions models were used to measure the association between the primary predictor variable (follow-up) and the primary outcome variable (stability) and other variables. Statistical significance was set at a $P$ value less than 0.05 .

\section{Results}

Eleven patients underwent SLF III and LF I osteotomies between September 2013 and February 2015. The sample consisted of six women (54.4\%) and 5 men with an average age of $23.83 \pm 4.17$ years and a mean negative overjet of $13.5 \pm 1.38 \mathrm{~mm}$. The mean advancement of the upper incisor immediately after surgery was $10.03 \pm 1.6 \mathrm{~mm}$. All patients underwent sagittal split osteotomy for mandibular rotations, and four also underwent sliding chin osteotomy advancement. Table 1 summarises the study sample, including all study variables (outcome variables) versus follow-up period (primary predictor variable), including descriptive statistics and bivariate analysis of primary surgical changes. Horizontal changes of the upper incisors, A-point, and posterior nasal spine were statistically significant.

Table 2 presents a summary of the bivariate analysis between primary predictor variable (follow-up) versus primary outcome variable (stability) measured with Mimics software. Table 3 presents a summary of the bivariate analysis between primary predictor variable (follow-up) versus primary outcome variable (stability) measured with Dolphin software. No statistical differences were observed between vertical and horizontal surgical changes immediately after surgery and 18 months after surgery. Statistically significant differences in horizontal landmark positions were noted only for PNS in the Mimics group, and the difference in millimetres between $\mathrm{T} 1$ and $\mathrm{T} 2$ ranged from $10.51 \pm 2.06$ to $9.35 \pm 2.10 \mathrm{~mm}$, respectively. 


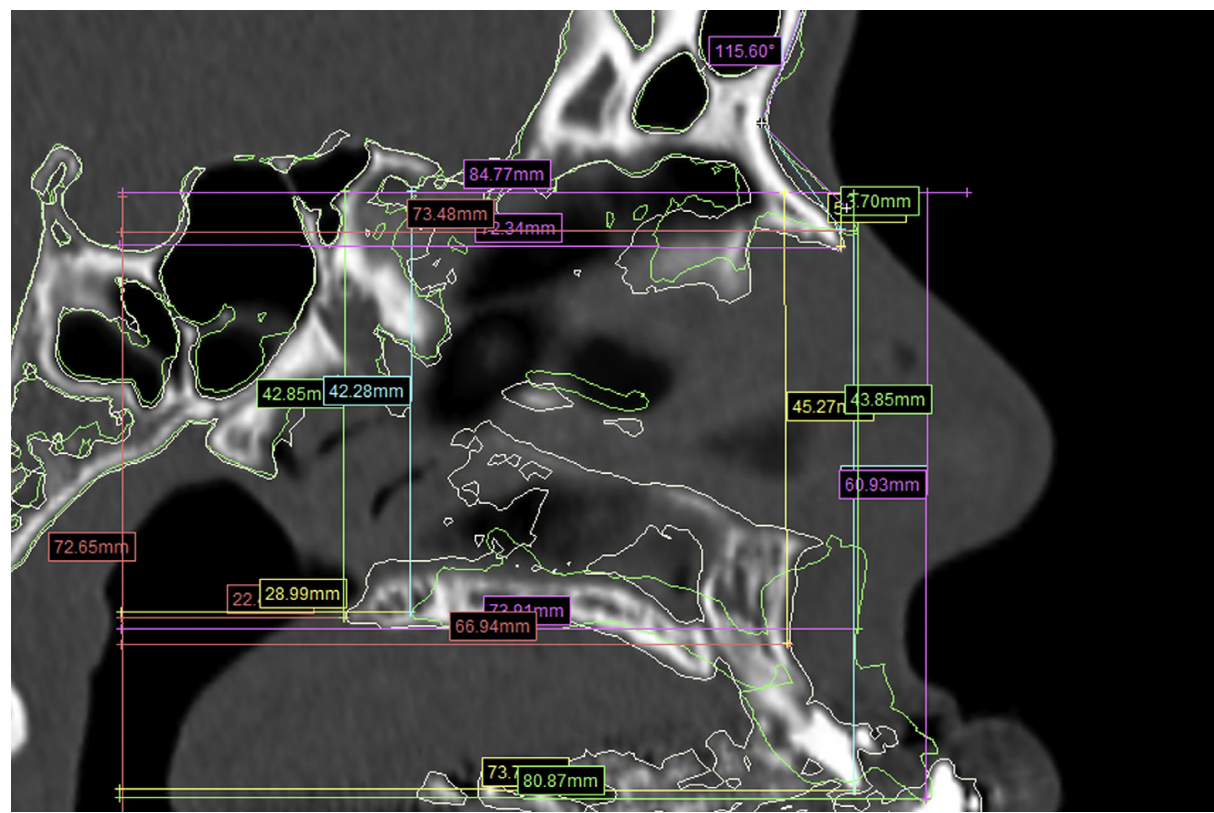

Fig. 3. Linear horizontal and vertical measures after registration and superimposition with Mimics software.

To determine whether there were differences between the two third-party software programs, the authors compared the primary outcome variable values between Mimics and Dolphin, for the immediately postoperative time point and 18 months after surgery. Table 4 presents the results of this bivariate analysis. Table 5 presents a comparison of primary outcome variables between Dolphin and Mimics software after 18 months from surgery. No statistical differences were observed between vertical and horizontal surgical changes immediately after surgery and 18 months after surgery. The horizontal and vertical differences of landmark positions after

Table 1

Summary of study variables (outcome variables) versus follow-up period (primary predictor variable), including descriptive statistics.

\begin{tabular}{lll}
\hline Study variable & Study group & $P$-value \\
\hline Sample size, n & 11 & - \\
Men, n (\%) & $5(45.6)$ & - \\
Age (yr), mean \pm SD & $23.83 \pm 4.17$ & - \\
Type of surgery, n (\%) & $11(100)$ & - \\
Le Fort III & $11(100)$ & - \\
Le Fort I & $7(63.6)$ & - \\
BSSO & $4(36.4)$ & - \\
Chin & & \\
Pre-surgical variables & $13.5 \pm 1.38$ & 0.004 \\
Overjet (mm), mean \pm SD & $132.84 \pm 5.77$ & 0.99 \\
Upper incisor inclination (degrees), mean \pm SD & & \\
Surgical changes (mm), mean \pm SD & $10.03 \pm 1.6$ & 0.05 \\
Upper incisor horizontal & $0.27 \pm 3.22$ & 0.90 \\
Upper incisor vertical & $10.98 \pm 2.25$ & 0.003 \\
A-point horizontal & $0.10 \pm 3.01$ & 0.91 \\
A-point vertical & $10.11 \pm 1.67$ & 0.002 \\
PNS horizontal & $0.85 \pm 3.16$ & 0.54 \\
PNS vertical & $4.77 \pm 1.41$ & 0.54 \\
Right infra-orbital horizontal & $0.26 \pm 2.39$ & 0.73 \\
Right infra-orbital vertical & $4.47 \pm 1.68$ & 0.70 \\
Left infra-orbital horizontal & $0.05 \pm 2.43$ & 0.95 \\
Left infra-orbital vertical & $2.87 \pm 1.39$ & 0.77 \\
Tip of nasal bone horizontal & $0.23 \pm 2.98$ & 0.64 \\
Tip of nasal bone vertical & 18 & - \\
Follow-up period (months) & 2 & - \\
Software for measurements (n) & & \\
\hline
\end{tabular}

Legends: LF III, Le Fort III osteotomy; LF I, Le Fort I osteotomy; BSSO, Bilateral Sagittal Split Osteotomy.
Table 2

Summary of bivariate association between primary predictor variable (follow-up) versus primary outcome variable (stability) measured with Mimics software.

\begin{tabular}{|c|c|c|c|c|c|}
\hline & \multicolumn{2}{|c|}{$\mathrm{T} 1-\mathrm{T} 0$} & \multicolumn{2}{|l|}{$\mathrm{T} 2-\mathrm{T} 0$} & \multirow[t]{2}{*}{$P$-value* } \\
\hline & Mean & SD & Mean & SD & \\
\hline \multicolumn{6}{|l|}{ Horizontal } \\
\hline Upper Incisor - Right & 10.01 & 1.92 & 10.18 & 2.35 & 0.72 \\
\hline PNS & 10.51 & 2.06 & 9.35 & 2.05 & 0.02 \\
\hline A-point & 10.14 & 2.50 & 9.41 & 2.44 & 0.32 \\
\hline Infra-Orbital - Right & 4.23 & 1.59 & 4.00 & 1.85 & 0.70 \\
\hline Infra-Orbital - Left & 3.86 & 2.04 & 3.63 & 1.44 & 0.68 \\
\hline \multicolumn{6}{|l|}{ Vertical } \\
\hline Upper Incisor - Right & 0.36 & 4.07 & 0.76 & 3.98 & 0.15 \\
\hline PNS & 1.76 & 3.66 & 1.12 & 3.27 & 0.35 \\
\hline A-point & 0.37 & 2.80 & 0.24 & 2.64 & 0.84 \\
\hline Infra-Orbital - Right & 0.91 & 1.36 & 0.46 & 2.97 & 0.83 \\
\hline Infra-Orbital - Left & 0.17 & 3.03 & 0.89 & 2.35 & 0.13 \\
\hline
\end{tabular}

T0, before surgery, T1, Immediate post-operative time; T2, after 18 months of surgery; PNS, Posterior nasal spine.

Values in $\mathrm{mm}$. *T-test.

Table 3

Summary of bivariate association between primary predictor variable (follow-up) versus primary outcome variable (stability) measured with Dolphin software.

\begin{tabular}{lllllll}
\hline & T1-T0 & & & T2-T0 & & P-value* \\
& Mean & SD & & Mean & SD & \\
\hline Horizontal & & & & & \\
$\quad$ Upper Incisor - Right & 9.88 & 2.44 & & 10.05 & 2.37 & 0.19 \\
$\quad$ PNS & 10.37 & 1.60 & 9.70 & 2.37 & 0.12 \\
A-point & 11.25 & 3.14 & 9.63 & 2.91 & 0.13 \\
$\quad$ Infra-Orbital - Right & 4.43 & 1.48 & 4.45 & 1.65 & 0.94 \\
$\quad$ Infra-Orbital - Left & 2.25 & 3.13 & 2.12 & 2.96 & 0.22 \\
Vertical & & & & & \\
$\quad$ Upper Incisor - Right & 0.45 & 4.23 & 0.28 & 2.30 & 0.58 \\
$\quad$ PNS & 0.07 & 3.25 & 1.28 & 2.94 & 0.17 \\
A-point & 0.37 & 5.06 & 1.43 & 3.64 & 0.38 \\
$\quad$ Infra-Orbital - Right & 0.93 & 2.34 & 0.80 & 2.65 & 0.83 \\
$\quad$ Infra-Orbital - Left & 0.15 & 1.67 & 0.10 & 1.66 & 0.62 \\
\hline
\end{tabular}

T0, before surgery, T1, Immediate post-operative time; T2, after 18 months of surgery; PNS, Posterior nasal spine.

Values in $\mathrm{mm}$. ${ }^{*}$ T-test. 
Table 4

Comparison of primary outcome variables between dolphin and mimics software during immediate postoperative period.

\begin{tabular}{llll}
\hline & Dolphin & Mimics & $P$ value* \\
\hline Upper incisor horizontal & $9.89 \pm 1.89$ & $10.03 \pm 1.60$ & 0.81 \\
Upper incisor vertical & $0.78 \pm 3.07$ & $-0.27 \pm 3.22$ & 0.25 \\
A-point horizontal & $10.48 \pm 2.07$ & $10.98 \pm 2.25$ & 0.81 \\
A-point vertical & $0.15 \pm 4.18$ & $0.10 \pm 3.01$ & 0.86 \\
PNS horizontal & $10.11 \pm 1.39$ & $10.11 \pm 1.67$ & 0.92 \\
PNS vertical & $0.36 \pm 2.71$ & $-0.85 \pm 3.16$ & 0.29 \\
Right infra-orbital horizontal & $4.80 \pm 1.40$ & $4.77 \pm 1.41$ & 0.81 \\
Right infra-orbital vertical & $0.62 \pm 2.47$ & $0.26 \pm 2.49$ & 0.62 \\
Left infra-orbital horizontal & $3.69 \pm 2.82$ & $4.47 \pm 1.68$ & 0.53 \\
Left infra-orbital vertical & $-0.03 \pm 1.51$ & $0.05 \pm 2.43$ & 0.74 \\
Tip of nasal bone horizontal & $3.56 \pm 1.66$ & $2.87 \pm 1.39$ & 0.41 \\
Tip of nasal bone vertical & $0.55 \pm 1.72$ & $-0.23 \pm 2.98$ & 0.62 \\
\hline
\end{tabular}

*Mann-Whitney (Wilcoxon Rank-Sum test).

Table 5

Comparison of primary outcome variables between dolphin and mimics software after 18 months of surgery.

\begin{tabular}{llll}
\hline & Dolphin & Mimics & $P$ value* \\
\hline Upper incisor horizontal & $10.67 \pm 2.37$ & $10.54 \pm 1.97$ & 0.97 \\
Upper incisor vertical & $0.26 \pm 1.84$ & $-0.37 \pm 3.03$ & 0.94 \\
A-point horizontal & $9.06 \pm 3.04$ & $9.71 \pm 1.87$ & 0.97 \\
A-point vertical & $-0.75 \pm 3.13$ & $-0.13 \pm 2.74$ & 0.76 \\
PNS horizontal & $9.86 \pm 1.84$ & $9.81 \pm 1.68$ & 0.99 \\
PNS vertical & $-0.81 \pm 2.46$ & $-0.72 \pm 2.68$ & 0.76 \\
Right infra-orbital horizontal & $4.98 \pm 1.56$ & $4.94 \pm 1.81$ & 0.92 \\
Right infra-orbital vertical & $0.70 \pm 2.57$ & $1.01 \pm 2.63$ & 0.97 \\
Left infra-orbital horizontal & $3.84 \pm 2.95$ & $4.70 \pm 1.69$ & 0.57 \\
Left infra-orbital vertical & $0.25 \pm 1.64$ & $-0.29 \pm 2.26$ & 0.69 \\
Tip of nasal bone horizontal & $3.83 \pm 1.40$ & $3.71 \pm 1.52$ & 0.97 \\
Tip of nasal bone vertical & $-0.17 \pm 1.45$ & $0.19 \pm 2.04$ & 0.81 \\
\hline
\end{tabular}

*Mann-Whitney (Wilcoxon Rank-Sum test).
18 months of surgery were less than $0.01 \mathrm{~mm}$. Fig. 4 shows the stable result of the SLF III plus LF I using a colour map of superimposition.

Table 6 presents the regression model that assessed the influence of age, gender, overjet, upper incisor angle, and genioplasty as potential effect modifiers of the primary outcome variable results. The regression analysis showed that SLF III and LF I osteotomies have a positive association with stability in large overjets (coefficient $=0.7 ; 95 \%$ confidence interval, $0.08-1.49 ; P=0.03$ ).

\section{Discussion}

The purpose of this study was to evaluate the stability of a series of non-syndromic patients who underwent SLF III osteotomy with LF I osteotomy after 18 months of surgery. The authors hypothesised that the combination of these two techniques would yield a clinically stable result. The specific aims of this study were to measure and compare 1) the vertical and horizontal movements of reproducible cephalometric landmarks immediately after and 18 months after surgery and 2) compare the measures obtained by two different third-party software programs.

The results of this study confirmed the authors' hypothesis that SLF III plus LF I is a stable procedure to treat large class III malocclusions associated with midface hypodevelopment. There were no statistical differences between cephalometric landmark measures immediately after and 18 months after surgery. The regression analysis showed that SLF III and LF I osteotomies have a positive association with stability in large overjets (coefficient $=0.7 ; 95 \%$ confidence interval, $0.08-1.49 ; P=0.03$ ). These findings allowed the authors to accept the hypothesis that the combination of sLF III and LF I was stable when treating large class III malocclusion patients with overjet greater than $10 \mathrm{~mm}$.

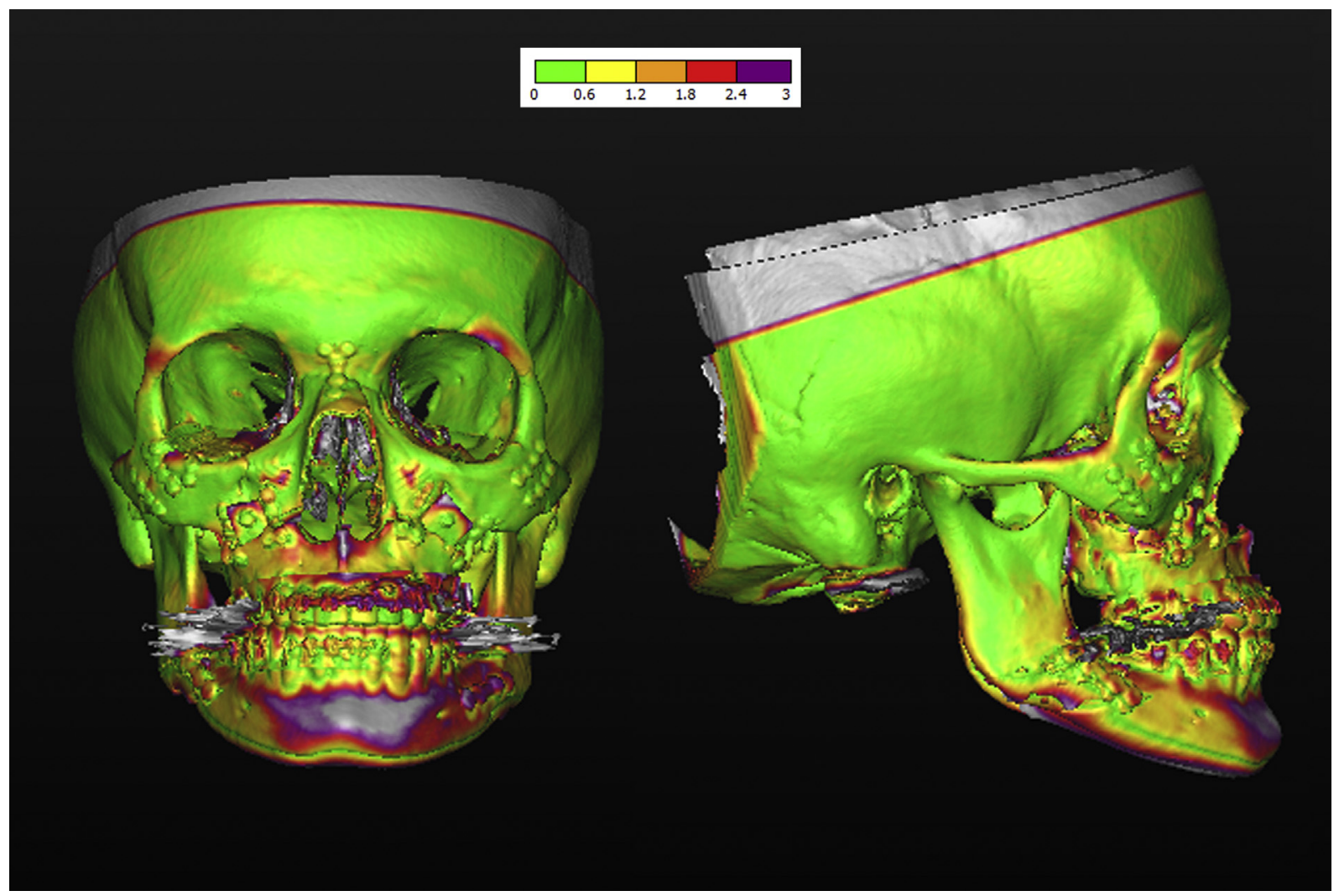

Fig. 4. Colour map of the superimposition between $\mathrm{T} 1$ and T2, showing the stability of the Le Fort III osteotomy. Green means variation less than 0.6 mm. 
Table 6

Regression models primary predictor variable (follow-up) versus outcome variables.

\begin{tabular}{llll}
\hline Study variable & Coefficient & Confidence interval (95\%) & $P$ value \\
\hline Age & 1.3 & $-1.60-4.22$ & 0.33 \\
Gender & -0.2 & $-0.60-1.60$ & 0.21 \\
Overjet & 0.7 & $0.08-1.49$ & 0.03 \\
UI Angle & -0.09 & $-6.75-6.56$ & 0.97 \\
Chin & -0.16 & $-0.56-0.24$ & 0.38 \\
\hline
\end{tabular}

UI Angle, Upper Incisor angle; BSSO, Bilateral Sagittal Split Osteotomy; Chin, Genioplasty.

Note: After adjusting for age, gender, overjet, UI angle, BSSO and Chin surgery, only the overjet was associated with stability after 18 months of surgery. This result suggests that performing LF III and LF I have a great postoperative stability.

Vertical and horizontal movements were stable after 18 months. The stability reported in this study may be associated with 1 ) the surgical technique, 2) the type of fixation, and 3) the final position of soft tissue after advancing the middle third of the face. Regarding the surgical technique, performing a horizontal osteotomy at the body of the zygoma and nasal bones during SLF III, the surgeon will achieve a large surface on which the midface will slide forward and in an upper direction under the patient's skull. This design will improve stability by increasing the contact and avoiding gaps between segments, thus avoiding the need for grafts. In addition, regarding the design of the zygoma body osteotomies, they should be parallel to each other. By failing to make them parallel after advancement, a differential contact may be created between sides, creating gaps and different heights of the zygoma, thus causing an asymmetry and the necessity of grafting one side of the osteotomy to fill the created gap. Considering the type of fixation, fixating the nasal bones helps avoid the rotation of the midface complex, favouring long-term stability. The zygoma osteotomies were fixed with two plates on each side. All patients also underwent sagittal osteotomy of the mandible instead of the vertical ramus osteotomy, avoiding a long period of intermaxillary fixation.

One may ask why the SLF III in combination with the LF I is so stable for larger horizontal movements, whereas the LF I osteotomy did not show this stability. A sound hypothesis is that, after a high pterigomaxillary disjunction, midface advancement will move forward all pharyngeal and palatal muscles that insert at the maxilla and will secure the skin in an advanced position. Therefore, after LF III advancement, the muscles that will hold the maxilla in a backward position are not yet stretched. Consequently, the maxilla at LF I osteotomy may be advanced $6-8 \mathrm{~mm}$ in addition to midface advancement, until the muscles and skin are stretched. The bone contact and the use of stable internal fixation will also improve stability.

Dolphin software showed a tendency to round the numbers within one decimal of millimetres, whereas Mimics software tended to show two decimals of millimetres during measures. Both software programs used in this study had very similar results, with a range of mean difference less than $0.01 \mathrm{~mm}$, without statistical significance. Because both software programs were similar in performance, primary outcome variables of one software program could be interpreted as a positive control of the other.

There are a number of studies that concluded that the standard LF III procedure provides a relatively stable postoperative position of the midface, making this technique reliable and applicable for treating patients with midface hypoplasia (Bouw et al., 2015; Schmitz et al., 1995). First described by Cheung (Cheung et al., 2010), the sliding osteotomy that is done on the zygomatic bone bilaterally, in the LF III osteotomy, provides good bone contact between the bone segments, which are two great advantages of this technique. First, there is no need for bone graft in the midface advancement and second, the bone contact improves the stability of the LF III osteotomy, making relapse less probable.

The first report of a patient who had a midface osteotomy was published in 1950 (Gillies and Harrison, 1950). The authors observed that the position of the central incisor was stable after 7 years. Freihofer was the first to describe the cephalometric results of nine patients after LF III osteotomies, two of whom underwent simultaneous LF III and LF I osteotomies. The follow-up time points occurred at various intervals up to 3 years. Osteotomized segments were stabilised with bone grafts and intermaxillary fixation. The two patients with simultaneous LF III/LF I osteotomies had $3 \mathrm{~mm}$ and $4 \mathrm{~mm}$ of vertical maxillary relapse at soft-tissue nasion. The maxilla was relatively stable in the horizontal direction, with one patient experiencing $3 \mathrm{~mm}$ of inferior relapse (Freihofer, 1973).

Modifications and combinations of midface osteotomies exist to correct midface deformations, but only sLF III osteotomy addresses the nose, orbits, cheeks, and maxilla (Schmitz et al., 1995). Although functional, LF III osteotomy also improves facial aesthetics, including all the midface bones.

Compared to Cheung's subcranial sliding LF III technique (Cheung et al., 2010), a difference in our surgical protocol is the fixation on the nasal osteotomy. Fixating the nasal bones helps to avoid the rotation of the midface complex, favouring long-term stability. This anti-clockwise rotation is one the primary causes of relapse in the midface osteotomy (Schmitz et al., 1995).

Skeletal and occlusal relapse with midface osteotomies was also discussed by Epker and Wolford (1975). Their cases were stabilised using wires and bone grafts, and the occlusion was overcorrected in all cases. In addition, intermaxillary fixation was employed for 6-8 weeks, after which the fixation was removed and splinted with intermaxillary elastics for 2-4 weeks.

Proffit (Proffit et al., 2007) considers changes smaller than $2 \mathrm{~mm}$ to be within the range of method error and clinically insignificant; 2-4 $\mathrm{mm}$ changes are outside the range of method error and potentially clinically significant; and changes greater than $4 \mathrm{~mm}$ are often beyond the range of orthodontic compensation and clinically highly significant. The results of this study showed stability during a long follow-up period, within less than $2 \mathrm{~mm}$ of relapse after 18 months of treatment. Although the PNS Mimics group showed a statistical difference clinically, the mean difference is in the range of method error and clinically insignificant.

The weakness of this study was the technique used to register and superimpose the 3D structures of each patient, raising question on how precise the under millimetric measures are. The technique used in this study was landmark based. This technique is landmarkidentification dependent and may not be as precise as automated or manual voxel-based superimposition (Weissheimer et al., 2015). However, this study used two different third party software programs that provided very similar results, without statistical differences. Furthermore, the method of quantitative measure by semitransparent slices of anatomical planes proves to be correct, considering the two-way methods of analysis.

\section{Conclusion}

In conclusion, this study supports that SLF III midface advancement combined with LF I is effective in maintaining stable horizontal and vertical skeletal positioning after surgery in nonsyndromal patients. Further, both software programs showed similar results and proved to be an efficient clinical tool to study stability.

\section{Funding}

This research did not receive any specific grant from funding agencies in the public, commercial, or not-for-profit sectors. 


\section{References}

Bachmayer DI, Ross RB: Stability of Le Fort III advancement surgery in children with Crouzon's, Apert's, and Pfeiffer's syndromes. Cleft Palate J 23: 69-74, 1986

Bouw FP, Nout E, van Bezooijen JS, Koudstaal MJ, Veenland JF, Wolvius EB: Threedimensional position changes of the midface following Le Fort III advancement in syndromic craniosynostosis. J Craniomaxillofac Surg 43: 820-824. https:// doi.org/10.1016/j.jcms.2015.04.003, 2015

Cheung LK, Ow A, Chua HDP: Simultaneous modified oblique Le Fort III and segmentalized Le Fort I osteotomies. J Oral Maxillofac Surg 68: 915-923. https:// doi.org/10.1016/j.joms.2009.06.009, 2010

Epker BN, Wolford LM: Middle-third facial osteotomies: their use in the correction of acquired and developmental dentofacial and craniofacial deformities. J Oral Surg 33: 491-514, 1975

Freihofer Jr HP: Results after midface-osteotomies. J Maxillofac Surg 1: 30-36, 1973

Gillies H, Harrison SH: Operative correction by osteotomy of recurred malarmaxillary compound in a case of oxycephaly. Br J Plast Surg 3: 123-127, 1950

Guijarro-Martínez R, Swennen GR: Three-dimensional cone beam computed tomography definition of the anatomical subregions of the upper airway: a validation study. Int J Oral Maxillofac Surg 42: 1140-1149. https://doi.org/ 10.1016/j.ijom.2013.03.007, 2013

Kaban LB, Conover M, Mulliken JB: Midface position after Le Fort III advancement: a long-term follow-up study. Cleft Palate J 23: 75-77, 1986

McCarthy JG, La Trenta GS, Breitbart AS, Grayson BH, Bookstein FL: The Le Fort III advancement osteotomy in the child under 7 years of age. Plast Reconstr Surg 86: 633-646, 1990

Meazzini MC, Mazzoleni F, Caronni E, Bozzetti A: Le Fort III advancement osteotomy in the growing child affected by Crouzon's and Apert's syndromes: presurgica and postsurgical growth. J Craniofac Surg 16: 369-377, 2005

Nout E, Cesteleyn LLM, van der Wal KGH, van Adrichem LNA, Mathijssen IMJ Wolvius EB: Advancement of the midface, from conventional Le Fort II osteotomy to Le Fort III distraction: review of the literature. Int J Oral Maxillofac Surg 37: 781. https://doi.org/10.1016/j.ijom.2008.04.006, 2008
Ousterhout DK, Vargervik K, Clark S: Stability of the maxilla after Le Fort III advancement in craniosynostosis syndromes. Cleft Palate J 23: 91-101, 1986

Proffit WR, Turvey TA, Phillips C: The hierarchy of stability and predictability in orthognathic surgery with rigid fixation: an update and extension. Head Face Med 3: 21. https://doi.org/10.1186/1746-160X-3-21, 2007

Schmitz JP, Tiner BD, Van Sickels JE: Stability of simultaneous modified LeFort III/ LeFort I osteotomies. J Craniomaxillofac Surg 23: 287-295, 1995

Shetye PR, Davidson EH, Sorkin M, Grayson BH, McCarthy JG: Evaluation of three surgical techniques for advancement of the midface in growing children with syndromic craniosynostosis. Plast Reconstr Surg 126: 982-994. https://doi.org/ 10.1097/PRS.0b013e3181e6051e, 2010a

Shetye PR, Kapadia H, Grayson BH, McCarthy JG: A 10-year study of skeletal stability and growth of the midface following Le Fort III advancement in syndromic craniosynostosis. Plast Reconstr Surg 126: 973-981. https://doi.org/10.1097/ PRS.0b013e3181e60502, 2010b

Tessier P: Osteotomies totales de la face: syndrome de Crouzon, syndrome D'Aper. Oxycephalies, scapho-cephalies, turricephalies. Ann Chir Plast 12: 273-286, 1967

Tessier P: The definitive plastic surgical treatment of the severe facial deformities of craniofacial dysostosis: Crouzon and Apert diseases. Plast Reconstr Surg 48: 419-442, 1971a

Tessier P: Total osteotomy of the middle third of the face for faciostenosis or for sequelae of Le Fort 3 fracture. Plast Reconstr Surg 48: 533-541, 1971b

Tiwana PS, Turvey TA, Ruiz RL: Long-term stability of subcranial Le Fort III osteotomy in syndromic and non-syndromic patients. J Oral Maxillofac Surg 58: 50-51, 2000

Tiwana PS, Turvey TA: Subcranial procedures in craniofacial surgery: the Le Fort III osteotomy. Oral Maxillofac Surg Clin N Am 16: 493, 2004

Weissheimer A, Menezes LM, Koerich L, Pham J, Cevidanes LHS: Fast threedimensional superimposition of cone beam computed tomography for orthopaedics and orthognathic surgery evaluation. Int J Oral Maxillofac Surg 44: 1188-1196. https://doi.org/10.1016/j.ijom.2015.04.001, 2015 